\title{
Preliminary Experiment Combining Virtual Reality Haptic Shoes And Audio Synthesis
}

\author{
Rolf Nordahl ${ }^{1}$, Amir Berrezag ${ }^{2}$, Smilen Dimitrov ${ }^{1}$, \\ Luca Turchet ${ }^{1}$, Vincent Hayward ${ }^{2}$ and Stefania Serafin ${ }^{1}$ \\ 1 Aalborg University Copenhagen, Medialogy, Lautrupvang 15, 2750 \\ Ballerup, Denmark \{rn, sd, tur, sts $\}$ @media.aau.dk \\ 2 UPMC Univ Paris 06, Institut des Systèmes Intelligents et de Robotique, 4 place \\ Jussieu, 75005 Paris, France \{amir.berrezag, vincent.hayward\}@isir.upmc.fr
}

\begin{abstract}
We describe a system that can provide combined auditory and haptic sensations that arise while walking on different grounds. The simulation is based on a physical model that drives both haptic transducers embedded in sandals and headphones. The model is able to represent walking interactions with solid surfaces that can creak, be covered with crumpling material. The simulation responds to pressure on the floor by a vibrotactile signal felt by the feet. In a preliminary discrimination experiment, 15 participants were asked to recognize four different surfaces in a list of sixteen possibilities and under three different conditions, haptics only, audition only and combined haptic-audition. The results indicate that subjects are able to recognize most of the stimuli in the audition only condition, and some of the material properties such as hardness in the haptics only condition. The combination of auditory and haptic cues does not significantly improve recognition.
\end{abstract}

Key words: physical models, walking sounds, audio-haptic interaction.

\section{Introduction}

Multimodality is an increasingly common feature of interactive systems. Whilst most studies focus on the interaction between vision and audition or between vision and touch, interaction between touch and audition is also strong because of two sources of sensory information have high temporal resolution. The perception literature contains many reports of audio-tactile interaction effects, see [15, 14, $21,4]$ for examples and surveys, and there has been studies directed at leveraging audiotactile to enhance interaction with virtual worlds $[19,7,6,17,22,1]$.

We typically spend a great amount of our waking hours interacting with the world through our feet, performing simultaneous auditory and haptic probing, yet, most studies, so far, both from the perception and from the virtual reality literature, have focused on the hands. A notable exception is the work of Giordano et al., who showed that the feet were also effective at probing the world with discriminative touch, with and without access to auditory information. Their results suggested that integration of foot-haptic and auditory information did not follow simple integration rules [10]. 
Almost all haptic device development research is directed at stimulating the hand but what about the foot? Similarly to research on haptic devices for the hand, the approaches to stimulate the foot broadly follow two directions [11]. The force-feedback option has been explored considering either rolling or groundreferenced devices, e.g., [13,20]. This approach typically involve considerable engineering challenges and cost. The vibrotactile option has also been explored. There are two possible ways to provide the signal. The actuators can be embedded in the floor, see [23] for a survey, or they can be embedded in worn shoes. In the later case, however, to our knowledge, there has been no attempt up until now to aim for any kind of reproduction fidelity needed for a virtual reality simulation. Only signaling functions were considered $[9,16]$.

We describe a preliminary study carried out with haptic feedback sandals that employed a newly introduced broadband vibrotactile transducer. The transducers as well as the headphone worn by the participants were driven by the same physical model. The model, presented in greater detail elsewhere [18], is able to represent, for virtual reality purposes, the kind of interaction that one might expect from stepping on solid surfaces that may or may not present the characteritics of a creaking material or be covered by crumpling objects such as dry leaves. Within the auditory modality, this model was already shown to enable good discrimination among these materials.

Here, we used the same model to drive the haptic simulations in order to investigate whether the experience of a virtual world may be enhanced by providing haptic feedback through the feet. In the present study, the participants passively received sensory information through touch and and audition which is not the condition in which the system is intended to be used ultimately. Even though sensorimotor coupling was inexistent, as if another person did the walking for the participants, interesting results were obtained.

\section{Simulation Hardware and Software}

\subsection{Haptic Hardware}

A pair of light-weight sandals was procured (Model Arpenaz-50, Decathlon, Villeneuve d'Ascq, France). This particular model has light, stiff foam soles that are easy to gouge and fashion. Two cavities were made in the tickness of the sole to accommodate two vibrotactile actuators (Haptuator, Tactile Labs Inc., DeuxMontagnes, Qc, Canada). These electromagnetic recoil-type actuators have an operational, linear bandwidth of $50-500 \mathrm{~Hz}$ and can provide up to $3 \mathrm{G}$ of acceleration when connected to light loads. As indicated in Fig. 1, one actuator was placed under the heel of the wearer and the other under the ball of the foot. There were bonded in place to ensure good transmission of the vibrations inside the soles. When activated, vibrations propagated far in the light, stiff foam. In the present configuration, the two actuators were driven by the same signal but could be activated separately to emphasize, for instance, the front or back activation, to strick a balance, or to realize other effects such as modulating different, back-front signals during heel-toe movements. 
The sole has force sensors intended to pick the foot-floor interaction force in order the drive the audio and haptic synthesis. They were not used in the present study. As far as auditory feedback is concerned, it was delivered through closed headphones (DT 770, beyerdynamic, Heilbronn, Germany).

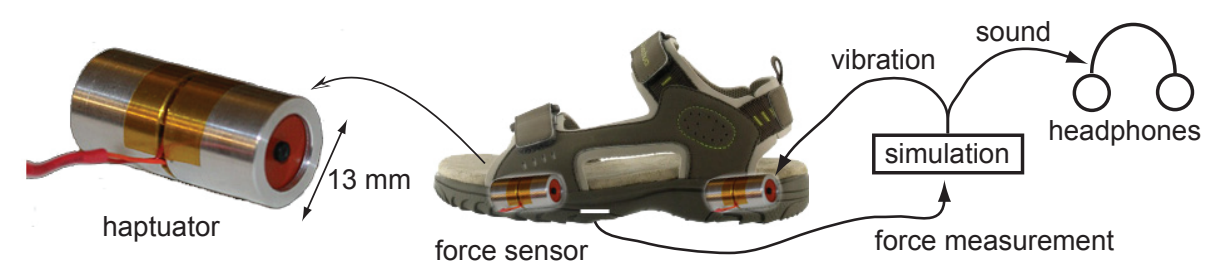

Fig. 1. System (one shoe shown). Left: recoil-type actuation from Tactile Labs Inc. The moving parts are protected by an alumimum enclosure able to bear the weight of a person. Middle: approximate location of the actuators in the sandal. Right: system diagram showing the interconnections. Here the force signal was not used.

\subsection{Audio-Haptic Simulation}

This model and its discretization is described elsewhere in detail [3]. The model has been recently adapted to the audio simulation of footsteps [18]. Here, we used the same model to drive the haptic and the audio synthesis. It is briefly recalled below.

A footstep sound may be considered to cause multiple micro-impacts between a sole, i.e., an exciter, and a floor, i.e., a resonator. Such interaction can be either discrete, as in the case of walking on a solid surface, or continuous, as in the case of a foot sliding across the floor.

In the simulation of discrete impacts, the excitation is brief and has an unbiased frequency response. The interaction is modelled by a Hunt-Crossley-type interaction where the force, $f$, between two bodies, combines hardening elasticity and a dissipation term [12]. Let $x$ represent contact interpenetration and $\alpha>1$ be a coefficient used to shape the nonlinear hardening, the special model form we used is

$$
f(x, \dot{x})=-k x^{\alpha}-\lambda x^{\alpha} \dot{x} \quad \text { if } x>0, \quad 0 \text { otherwise. }
$$

The model described was discretized as proposed in [2].

If the interaction called for slip, we adopted a model where the relationship between relative velocity $v$ of the bodies in contact and friction force $f$ is governed by a differential equation rather than a static map [8]. Considering that friction results from a large number of microscopic damped elastic bonds with an average deflection $z$, a viscous term, $\sigma_{2} v$, and a noise term, $\sigma_{3} w$, to represent roughness, we have

$$
f(z, \dot{z}, v, w)=\sigma_{0} z+\sigma_{1} \dot{z}+\sigma_{2} v+\sigma_{3} w .
$$


The force specified by these models is applied to a virtual mass which produces a displacement signal that is then processed by a linear shaping filter intended to represent the resonator.

Stochastic parameterization is employed to simulate particle interactions thereby avoiding to model each of many particles explicitely. Instead, the particles are assigned a probability to create an acoustic waveform. In the case of many particles, the interaction can be represented using a simple Poisson distribution, where the sound probability is constant at each time step, giving rise to an exponential probability weighting time between events.

We used this approach to model both solid and aggregate surfaces. A solid surface is represented by an impact and a slide. The impact model alone was used to recreate the sound and the feel produced when walking on wood. The friction model was tuned to simulate walking on creaking wood. To simulate walking on aggregate grounds, we used a physically informed sonic models (Phism) algorithm [5]. The synthesis was tuned to simulate snow and forest underbrush.

These algorithms were implemented as an extension to the Max/MSP platform $^{3}$ to drive both the auditory and haptic feedback.

\section{Preliminary Evaluation}

The experiment entailed asking three groups of fifteen participants to passively experience the stimuli described in the previous section. The first group received the haptic stimuli only, the second the audio only, and the third the combined stimuli. The synthesis was tuned to evoke four different surfaces: wood, creaking wood, snow, and underbrush. Participants had to select in a list of sixteen different materials one that matched best their experience. They also rated the realism and quality of their experience on a seven-point Likert scale.

Procedure: The participants were asked to wear the sandals, the headphones described in the previous section, and to sit in a chair. In the condition with haptics only they wore earplugs and sound protection headsets. Participants were asked to recognize the stimuli they were exposed to. They were given a list of sixteen materials: wood, creaking wood, underbrush, snow, frozen snow, beach sand, gravel, metal, "don't know", high grass, dry leaves, concrete, dirt, puddles, water, and carpet. Each of the four standard surfaces were presented twice in a randomized order. When presented one of the four standard stimuli, participants had to match it to one in the list and rated the realism and quality of the simulations. At the conclusion of the experiment, participants were asked to leave comments.

Participants: The forty five volunteers (students and faculty at the Engineering college of Copenhagen; 31 men and 14 women; average age 24.5 years, $\mathrm{sd}=4.6$ ) were randomly assigned to one of the three groups (audio only, haptic only or audio-haptic) for a total of 15 participants per condition. None reported hearing problems or other sensory impairments and wore shoes sizes from 41 and 45 .

\footnotetext{
${ }^{3}$ www.cycling74.com
} 
Results and discussion: Table 1 shows the confusion matrices produced by the three groups.

Table 1. Confusion matrices with: haptics, audio, and combined haptics and audio.

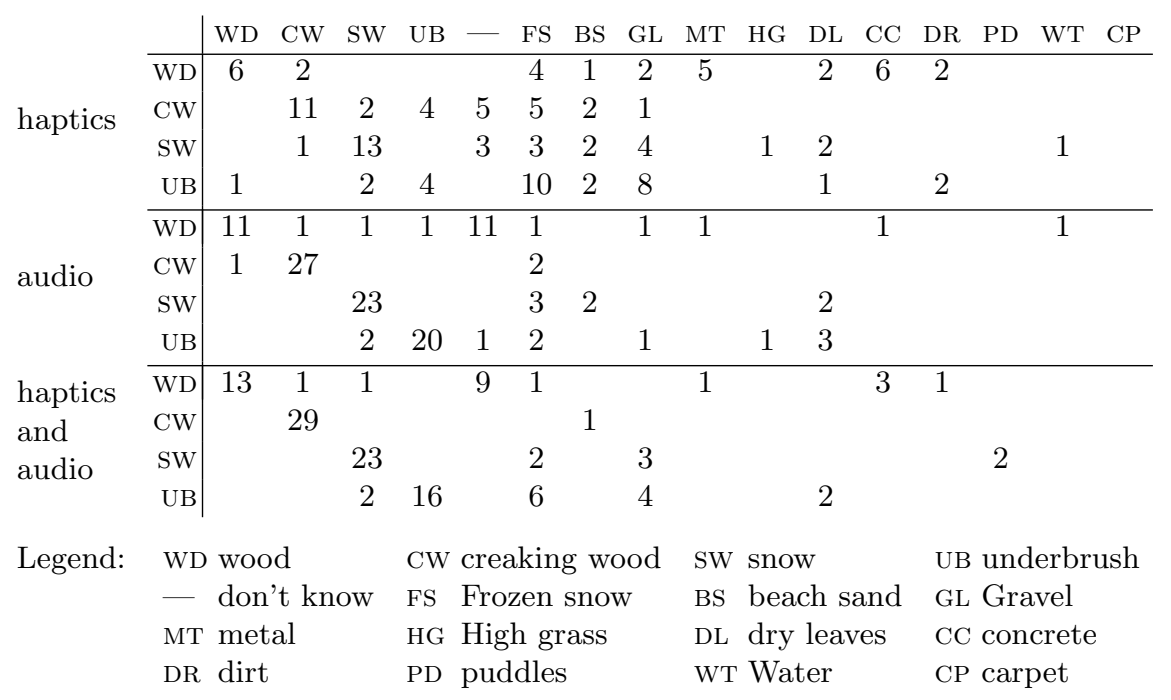

From the results, it can be noticed that haptic cues alone gave the subjects the possiblity to recognize surfaces categories but with poor fine discrimination. Such is the case for the wood simulation that was easily confused with metal, concrete or dirt. A solid surface was not confused with aggregates such as snow, underbrush, grass or with soft surfaces such as puddles or carpet. Recognition rates with audio stimuli were much better as seen in the corresponding dominant diagonal. The audio stimulation of wood still caused some confusion as seen in the numerous 'don't know' answers that was not present with the haptic experience. The small number of confusions was mostly among the aggregates. When the two stimuli (identical at the signal level) were delivered simultaneously, performance was not necessarily better as if conflict was created; a phenomenon which is noticeable during the presentation of simulated underbrush. What is quite interesting is that a similar phenomenon occurs during the multimodal identification of real materials [10] (full report forthcoming). In both haptic and auditory modality, the friction simulation was an important cue which facilitated the recognition of creaking wood.

Table 2 shows the degree to which participants judged the realism and quality of the experience. The degree of realism was calculated by looking only at that data from correct answers, i.e., when the surfaces were correctly recognized. As far as the quality judgement is concerned, the data was based on all the answers different from 'don't know'. 
Table 2. Average realism and quality scores from a seven-point Likert scale.

\begin{tabular}{|c|c|c|c|c|c|c|c|c|}
\hline & wood & eakir & $\begin{array}{l}\text { alisn } \\
\text { snow }\end{array}$ & lerbrush & & eakir & $\begin{array}{l}\text { ality } \\
\text { now }\end{array}$ & lerbrush \\
\hline haptics & 3.3 & 4.3 & 4.8 & 3.2 & 2.8 & 4.0 & 4.2 & 4.3 \\
\hline audio & 1.8 & 3.5 & 5.2 & 5.5 & 3.2 & 3.8 & 5.1 & 4.9 \\
\hline combined & 3.3 & 3.6 & 5.3 & 4.6 & 4.0 & 4.7 & 5.2 & 4.6 \\
\hline
\end{tabular}

\section{Conclusions and future work}

We described a system able to simulate the auditory and haptic sensation of walking on different materials and presented the results of a preliminary surface recognition experiment. This experiment was conducted under three different conditions: auditory feedback, haptic feedback, and both.

By presenting the stimuli to the participants passively sitting in a chair, we introduced a hight degree of control on the stimulation. However this method of delivery is highly contrived since it eliminates the tight sensorimotor coupling that is natural during walking and foot interaction. It is true for the auditory channel, but even more so for the haptic channel. In spite of these drasticly constrained conditiona, performance was surprisingly good.

We are currently running follow up experiments allowing subjects to walk in a controlled laboratory, where their steps are tracked and used to drive the simulation. We believe introducing a higher level of interactivity will significantly enhance the recognition rates as well as the perceived quality and realism of the simulation.

Acknowledgments. The research leading to these results has received funding from the European Community 's Seventh Framework Programme under FETOpen grant agreement n. 222107 NIW - Natural Interactive Walking.

\section{References}

1. F. Avanzini and P. Crosato. Integrating physically based sound models in a multimodal rendering architecture. The Journal of Visualization and Computer Animation, 17(3-4):411-419, 2006.

2. F. Avanzini and D. Rocchesso. Modeling collision sounds: Non-linear contact force. In Proc. COST-G6 Conf. Digital Audio Effects (DAFx-01), pages 61-66, 2001.

3. F. Avanzini, S. Serafin, and D. Rocchesso. Interactive simulation of rigid body interaction with friction-induced sound generation. IEEE transactions on speech and audio processing, 13(5 Part 2):1073-1081, 2005.

4. J.-P. Bresciani, M. O. Ernst, K. Drewing, G. Bouyer, V. Maury, and A. Kheddar. Feeling what you hear: Auditory signals can modulate tactile tap perception. Experimental Brain Research, 162:172-180, 2005.

5. P.R. Cook. Physically Informed Sonic Modeling (PhISM): Synthesis of Percussive Sounds. Computer Music Journal, 21(3):38-49, 1997. 
6. D. DiFilippo and D. K. Pai. Contact interaction with integrated audio and haptics. In Proceedings of the International Conference on Auditory Display, ICAD, 2000.

7. D.E. DiFranco, G. Lee Beauregard, and M.A. Srinivasan. The effect of auditory cues on the haptic perception of stiffness in virtual environments. Proceedings of the ASME Dynamic Systems and Control Division., 1997.

8. P. Dupont, V. Hayward, B. Armstrong, and F. Altpeter. Single state elastoplastic friction models. IEEE Transactions on Automatic Control, 47(5):787-792, 2002.

9. X. Fu and D. Li. Haptic shoes: representing information by vibration. In Proceedings of the 2005 Asia-Pacific symposium on Information visualisation, pages 47-50, 2005.

10. B. L. Giordano, S. Mcadams, Y. Visell, J. Cooperstock, H. Y. Yao, and V. Hayward. Non-visual identification of walking grounds. Journal of the Acoustical Society of America, 123(5):3412-3412, 2008.

11. V. Hayward and K. E. MacLean. Do it yourself haptics, part-i. IEEE Robotics and Automation Magazine, 14(4):88-104, 2007.

12. K. H. Hunt and F. R. E. Crossley. Coefficient of restitution interpreted as damping in vibroimpact. ASME Journal of Applied Mechanics, 42(2):440-445, 1975.

13. H. Iwata, H. Yano, and H. Tomioka. Powered shoes. In ACM SIGGRAPH 2006 Emerging technologies, page 28, 2006.

14. V. Jousmaki and R. Hari. Parchment-skin illusion: sound-biased touch. Current Biology, 8(6):R190-R191, 1998.

15. S.J. Lederman. Auditory texture perception. Perception, 1979.

16. M. Magana and R. Velazquez. On-shoe tactile display. In IEEE International Workshop on Haptic Audio visual Environments and Games (HAVE 2008), pages 114-119, 2008.

17. D.K. Pai, K. Doel, D.L. James, J. Lang, J.E. Lloyd, J.L. Richmond, and S.H. Yau. Scanning physical interaction behavior of $3 \mathrm{~d}$ objects. In Proceedings of the 28th annual conference on Computer graphics and interactive techniques, pages 87-96, 2001.

18. S. Serafin R. Nordahl and L. Turchet. Sound synthesis and evaluation of interactive footsteps for virtual reality applications. In Proc. IEEE VR 2010, 2010.

19. C. Ramstein and V. Hayward. The pantograph: A large workspace haptic device for a multi-modal human-computer interaction. In Proceedings of the SIGCHI conference on Human factors in computing systems, CHI'04, ACM/SIGCHI Companion4/94, pages 57-58, 1994.

20. H. Schmidt, S. Hesse, R. Bernhardt, and J. Krüger. Hapticwalker - a novel haptic foot device. ACM Transactions on Applied Perception, 2(2):166-180, 2005.

21. S. Shimojo and L. Shams. Sensory modalities are not separate modalities: plasticity and interactions. Current Opinion in Neurobiology, 11(4):505-509, 2001.

22. J. Sreng, F. Bergez, J. Legarrec, A. Lécuyer, and C. Andriot. Using an eventbased approach to improve the multimodal rendering of 6 dof virtual contact. In Proceedings of ACM Symposium on Virtual Reality Software and Technology (ACM VRST), pages 173-179, 2007.

23. Y. Visell, A. Law, and J. R. Cooperstock. Touch is everywhere: Floor surfaces as ambient haptic interfaces. IEEE Transactions on Haptics, 2:148-159, 2009. 